\title{
Evaluation of Prognostic Factors of Mortality in Patients with Chronic Obstructive Pulmonary Disease
}

\author{
Mehdi Torabi ${ }^{1}$ (D), Mitra Samareh Fekri ${ }^{2,(D)}$, Sara Azizi Shoul 1*iD, \\ Ahmad Naghibzadeh Thahami ${ }^{3 *(D)}$, Shiva Moeinaddini ${ }^{* *}$ (D)
}

1. Dept. of Emergency Medicine, Faculty of Medicine, Kerman University of Medical Sciences, Kerman, Iran

2. Pulmonology Cardiovascular Research Center, Institute of Basic and Clinical Physiology Sciences, Kerman University of Medical Sciences, Kerman, Iran

3. Dept. Epidemiology, Research Center for Modeling in Health, Institute for Future Studies in Health, Kerman University of Medical Sciences, Kerman, Iran

4. Dept. of Emergency Medicine, Faculty of Medicine, Rafsanjan University of Medical Sciences, Kerman, Iran

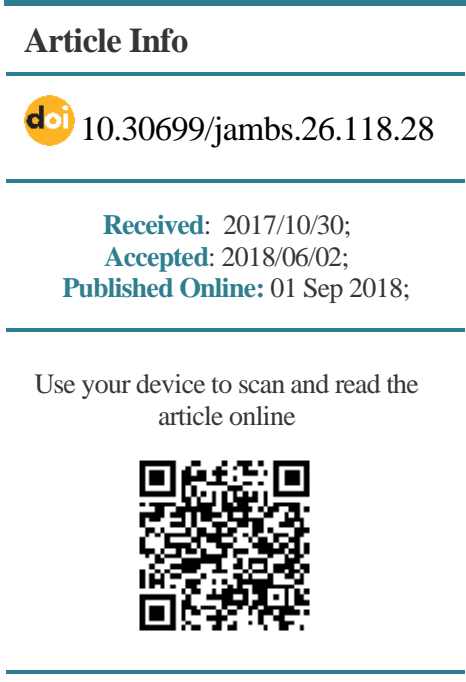

Corresponding Information: Mehdi Torabi,

Dept. of Emergency Medicine, Faculty of Medicine, Kerman University of Medical Sciences, Kerman, Iran Email:

me_torabi@kmu.ac.ir

\begin{abstract}
Background \& Objective: Chronic Obstructive Pulmonary Disease (COPD) is a chronic obstructive and irreversible disease which has a high mortality and morbidity rate. Systemic inflammation and the thrombotic process can influence the prognosis of these patients. The objectives of this study were to evaluate prognostic effects of CBC indices (WBC, PMN, MPV, RDW), forced expiratory volume-one second (FEV1) and mean Pulmonary Artery Pressure (mPAP) in the prognosis of COPD patients with acute exacerbation of the disease.

Materials \& Methods: This cross-sectional study was performed on exacerbated COPD patients who were admitted to the emergency department, Afzalipour Hospital, Kerman, Iran during 2016-2017. For all the patients, CBC was assessed as they arrived then Pulmonary Function Test and echocardiography were conducted. In order to create the final model, we employed multivariate regression analysis.

Results: A total of 1078 patients were enrolled during one year, of which $58.3 \%$ were male. In multivariate analysis, White blood cells (WBC), Polymorphonuclear leukocytes (PMN), Mean platelet volume (MPV), Red Cell Distribution Width (RDW), FEV1 and mPAP were the six variables which were independently associated with hospital mortality and ICU admission. Sensitivity, specificity and area under the curve for these six variables model were $78.5 \%, 92 \%$ and $86 \%$ respectively.
\end{abstract}

Conclusion: Since the inflammatory and thrombotic events are influential in the prognosis of COPD patients, it may be possible to predict patients outcome with $\mathrm{CBC}$ related indices (WBC, PMN, MPV and RDW), although other important risk factors such as pulmonary hypertension and FEV1 decrease should be considered as well.

Keywords: COPD, Mean platelet volume, Red Cell Distribution Width, Hospital mortality

\section{Introduction}

Chronic Obstructive Pulmonary Disease (COPD) is an irreversible inflammation of bronchial airways which results in parenchymal involvement as well as mucociliary malfunction. This disease is found in $5 \%$ of adults and it is responsible for one-fourth of death. It has been estimated that by the year 2020, COPD will be the third cause of death worldwide and a key factor of a society's health and hygiene due to the patient's repeated hospitalization requirements during exacerbation attacks owing to bacterial colonization in the lower parts of the respiratory system. This disease possesses a high mortality and morbidity rate and several factors involve in patients outcome, such that the role of inflammatory and thrombotic factors of the disease are not negligible (1-3).
White blood cells, especially neutrophils, which indicate the severity of inflammation in COPD patients, do not have a certain count and change in number throughout the stable and exacerbated phases of the disease. This is the result of the change of inflammation severity throughout different phases of the disease which can have a significant effect on the duration of the disease and the pulmonary function (4).

The mean platelet volume (MPV) is an indicator of platelet size, function, and activity. The larger platelets have dense granules which produce more serotonin, Bthromboglobulin and thromboxane A2 compared to the smaller types. Therefore, an increase of this marker indicates a higher risk of thrombotic events $(5,6)$. Furthermore, we are aware of the role of platelets in the inflammation process. The 
size of platelets present in the blood circulation and MPV are different, based on the severity of inflammation. In severe inflammatory conditions such as rheumatoid arthritis, MPV decreases due to the fact that only small platelets are in the blood circulation while the larger platelets are consumed at the inflammation site. In cases where there is less inflammation, such as psoriasis, MPV increases. As a result, the change in MPV can indicate both pre-inflammatory and pre-thrombotic activities (7-9). Although several studies have been conducted, the role of MPV in the outcome of COPD patients is not yet known and there have been inconsistent reports of the results of MPV changes (10-12).

Red Cell Distribution of Width (RDW) indicates the difference in the size of red blood cells or anisocytosis. In cases of inflammation such as COPD, RDW increases due to inhibition of erythropoiesis (13). This laboratory test which is proportional to the neutrophil-tolymphocyte ratio can have a prognostic role in determining the outcome and mortality rate of these patients (14-17). Also, its increase in COPD patients can demonstrate failure in the right ventricle (18).

The aim of this study was to evaluate $\mathrm{CBC}$ related tests (WBC, PMN, MPV, RDW) besides mean pulmonary artery pressure (mPAP) and forced expiratory volume-one second (FEV1) in COPD exacerbated patients in order to determine the association of each test with patients' outcome. In the present study, the patient's outcomes were hospital mortality (primary outcome), and also the need for ICU admission (secondary outcome). We tried to answer this question: Were these paraclinical parameters associated with mortality or ICU admission?

\section{Materials and Methods}

This cross-sectional study was done at the Kerman Afzalipour Hospital, in the southeast of Iran on patients with exacerbated COPD referred to the emergency department from June 21, 2016, through June 21, 2017. At the beginning stages, before initiating the treatment, Complete Blood Count (CBC) tests were conducted on all the patients less than one hour. When the patients were stabilized after receiving treatment protocol for COPD patients, pulmonary function test (PFT) and echocardiography for measurement of ejection fraction (EF) and mean pulmonary artery pressure (mPAP) were done. The inclusion criteria for the study was all COPD exacerbation patients (sudden worsening of shortness of breath, quantity, and color of sputum) whose airway obstruction had an FEV1/FVC ratio less than 0.7 based on spirometric results (less than $12 \%$ or $200 \mathrm{cc}$ increase in FEV1 after 15 minute receiving 2 puffs salbutamol) and rule out the other obstructive pulmonary diseases. Patients with increased inflammatory response or thrombotic problems such as deep vein thrombosis (DVT), pulmonary emboli, malignancies, febrile diseases, systemic hypertension, history of obstructive sleep apnea (OSA), renal failure, diabetes, left ventricular heart failure, patients being treated by antiinflammatory medications such as corticosteroids,
NSAIDs, ACEIs and statin drugs, patients with a history of antiplatelets or anti-coagulants therapy within the past two weeks, anemia, patients unwilling to cooperate, were excluded from the study (Figure 1).

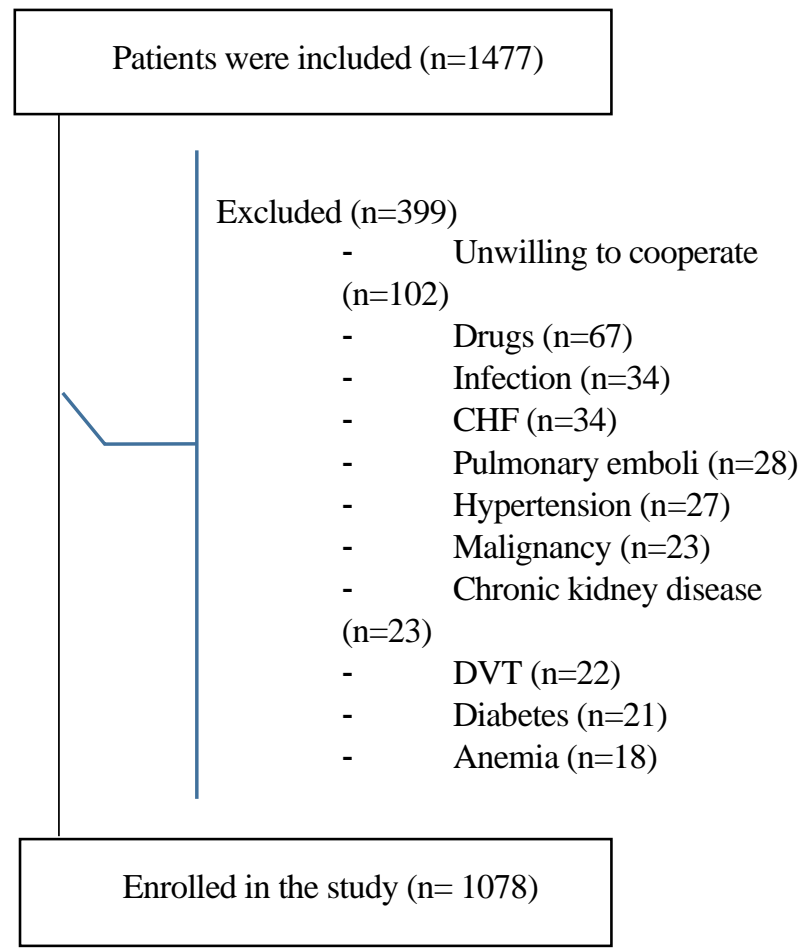

Figure 1. Flow chart showing enrollment of patients

CBC and its related tests (WBC, PMN, MPV, RDW) were collected blood in tubes that contained potassium citrate and tests were done less than an hour after the blood sampling. A pulmonary function test was done by an experienced technician for all patients in the respiratory room. Before doing the tests, the complete process was explained to the technician to perform the tests. On account of the obtained results, the severity of the disease was classified according to the Global Initiative for Chronic Obstructive Lung Disease (GOLD). A pulmonologist interpreted and approved the results. Besides, echocardiography was performed by Ejection Fraction (EF) and cardiologists to measure mean Pulmonary Artery Pressure (mPAP) to rule out the left ventricle heart failure in coronary care unit (CCU). An emergency medicine resident follow-up of the patients after diagnosis until the patients died or discharged. All of the data were obtained from handwritten files by an emergency medicine resident. An emergency medicine specialist frequently supervised data collection. Inhospital mortality and ICU admission were defined as the primary and secondary outcomes of this study, respectively. The hospital mortality was recorded by emergency medicine resident after checking hospital records. The ICU admission was done on the basis of predefined protocol and certified pulmonologist. This study followed the Helsinki Accords (1975), revised in Hong Kong (1989). The Ethics Committee of Kerman University of Medical Sciences approved the study 
(IR.KMU.REC 1394.566). We obtained informed consent from all patients before entering the study.

$\mathrm{CBC}$ and its related tests, FEV1, mPAP were the variables considered to be potentially correlated with the outcomes. These variables were considered quantitatively for their association with hospital mortality (primary outcome) and ICU admission (secondary outcome).

The description of quantitative variables was measured by Mean $( \pm S D)$, qualitative variables by frequency and severity of association by Odds ratio (OR) and 95\% confidence interval (CI). When p-value was less than 0.05 , this test was considered statistically significant. If $\mathrm{p}$-value was less than 0.25 in t-test and $\chi^{2}$ tests in univariate analysis, we were entered these variables in logistic regression model (19). In multivariate model, we used the backward conditional method. STATA version 12.0 (Stata Corp, College Station, TX, USA) was used for analysis.

\section{Results}

All quantitative variables that carried the potential possibility of hospital mortality and ICU admission were statistically analyzed by using univariate analysis. There was a significant difference between the variables and hospital mortality in this statistical model. Subsequently, Odds Ratio (OR) analysis was done to evaluate the severity of this association on the same variables (Table 2). For both univariate and multivariate analysis, MPV and RDW were both multiplied by 10 to evaluate its effects on the patient's outcome by a 0.1 increase or decrease since the change of 1 unit causes a great influence in the outcome and complicates obtaining of the results. For every 0.1 increase in MPV and RDW, there would be an 8 and $1 \%$ increase in-hospital mortality rate, respectively.

Table 1. Patients' characteristics in COPD patients

\begin{tabular}{ll} 
Variables & \multicolumn{1}{c}{$\mathbf{N}(\%)$} \\
Sex & \\
\hline Male & $628(58.26)$ \\
Female & $450(41.74)$ \\
GOLD staging & \\
\hline II & $442(41)$ \\
\hline III & $636(59)$ \\
Age $(y)$, Mean \pm SD & $70.13 \pm 12.26$ \\
\hline WBC, Mean \pm SD & $8362.05 \pm 4953.72$ \\
PMN, Mean \pm SD & $77.74 \pm 10.39$ \\
Hb, Mean \pm SD & $13.44 \pm 7.17$ \\
MPV, Mean \pm SD & $9.11 \pm 1.04$ \\
RDW, Mean \pm SD & $13.79 \pm 2.27$ \\
Plt, Mean \pm SD & $135630.4 \pm 80049.70$ \\
\hline
\end{tabular}

Volume 26, September \& October 2018

\begin{tabular}{lc} 
Variables & $\mathbf{N}(\%)$ \\
\hline mPAP(mmHg),Mean \pm SD & $37.22 \pm 7.29$ \\
$\mathrm{EF}(\%)$, Mean \pm SD & $54.92 \pm 5.97$ \\
\hline $\mathrm{FEV}{ }_{1}(\%)$, Mean \pm SD & $49.30 \pm 6.91$ \\
\hline
\end{tabular}

\begin{tabular}{ll}
\hline Outcome & \\
\hline A: Hospital mortality (\%) & $93(8.63)$ \\
\hline B: ICU Admission (\%) & $88(8.19)$
\end{tabular}

Abbreviation: WBC; White blood cell, PMN; Polymorphonuclear leukocytes, Hb; Hemoglobin, MPV; Mean platelet volume, RDW; Red cell distribution width, Plt; platelet, mPAP; mean pulmonary artery pressure, EF; Ejection fraction, FEVI; Forced expiratory volume-one second

Table 2. Univariate regression analysis of variables according to their association with mortality

\begin{tabular}{lll} 
Variable & Mortality & \\
& OR (95\% CI $)$ & P-value \\
Age & $1.00(0.99-1.02)$ & 0.37 \\
Sex & $1.00(0.65-1.55)$ & 0.96 \\
CBC & & \\
WBC & $1.01(1.01-1.02)$ & $<0.0001$ \\
PMN & $1.06(1.03-1.09)$ & $<0.0001$ \\
Hb & $1.11(1.02-1.20)$ & 0.01 \\
MPV*10 & $1.08(1.05-1.10)$ & $<0.0001$ \\
RDW*10 & $1.01(1.009-1.02)$ & $<0.0001$ \\
Plt & $1.00(0.99-1.01)$ & 0.08 \\
PFT & & \\
FE $V_{1}$ & $0.92(0.89-0.96)$ & $<0.0001$ \\
ECHOCAR & & \\
DIOGRAPHY & & $<0.0001$ \\
\hline mPAP & $1.05(1.03-1.08)$ & \\
\hline
\end{tabular}

Table 3. Multivariate regression analysis of variables according to their association with mortality

\begin{tabular}{lll} 
Variable & \multicolumn{2}{c}{ Mortality } \\
\hline OR(95\% CI) & P-value \\
PMN & $1.02(1.01-1.03)$ & $<0.0001$ \\
MPV*10 & $1.07(1.03-1.11)$ & $<0.0001$ \\
RDW $^{*} 10$ & $1.09(1.01-1.13)$ & $<0.0001$ \\
FEV & $1.02(1.01-1.03)$ & $<0.0001$ \\
mPAP & $0.89(0.85-0.94)$ & $<0.0001$ \\
\hline
\end{tabular}

Then multivariate model was done (backward conditional method). In this analysis, six variables (WBC, PMN, RDW, MPV, FEV1, mPAP) had a significant correlation with the hospital mortality rate: for every unit 
of increase in mPAP, the mortality rate increased by up to $11 \%$. Every 0.1 increase in MPV and RDW, increased the mortality rate by 9 and $2 \%$, respectively (Table 3 ).

The next step was the drawing of ROC curves by using logistic regression analysis on the six variables from the final model in order to determine the sensitivity and specificity to predict the hospital mortality and ICU admission. The results from the curve showed 0.86 AUC with a sensitivity and specificity of 78.5 and $92 \%$ for mortality rate (Figure 2). We also showed specificity, sensitivity, and AUC to determine the role of variables in the mortality rate (Table 4).

Furthermore, since the ICU admitted patients died, there was no difference between the two outcomes and therefore, the probability ratio of the first outcome (hospital mortality) was equal to the second outcome (ICU admission(

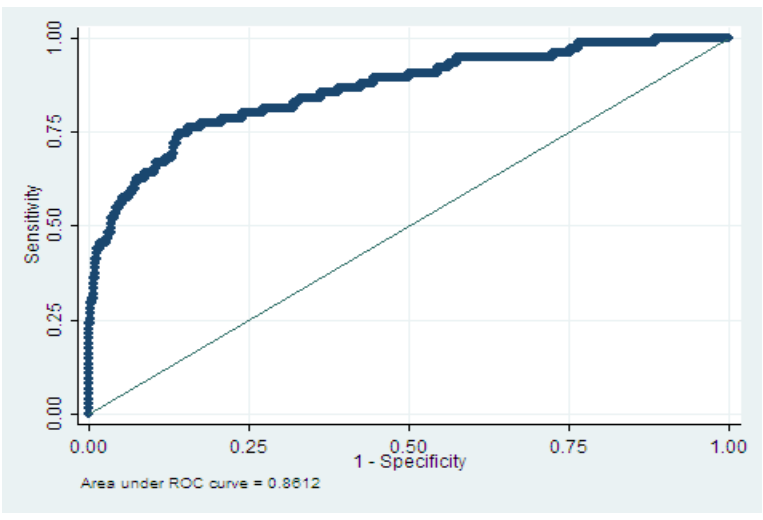

Figure 2. The ROC curve for mortality prediction by six variables.

Table 4. AUC, Sensitivity and Specificity of Models Created From the Final Model

\begin{tabular}{llll} 
& Sensitivity $(\%)$ & Specificity $(\%)$ & AUC \\
\hline TOTAL VARIABLES & 78.5 & 92 & 0.8612 \\
CBC(WBC+PMN+MPV+RDW) & 75 & 93 & 0.8095 \\
MPV & 68 & 92 & 0.7212 \\
RDW & 54.5 & 91 & 0.6206 \\
FEV1 & 58 & 91 & 0.6284 \\
mPAP & 52 & 91 & 0.5428 \\
\hline
\end{tabular}

\section{Discussion}

In this cross-sectional study, which was performed on COPD exacerbated patients referred to the emergency department for the period of one year, there was a significant correlation between CBC (WBC, PMN, MPV, RDW), mPAP and FEV1 with hospital mortality and ICU admission.

In the previous studies, MPV changes in COPD patients had different results. Biljak et al. Showed that MPV increase changes in stable COPD was an indicator of prethrombotic events or pulmonary hypertension (10). Ulasli et al. showed that in cases of exacerbation, MPV deceased and indicated a severe inflammatory process and negative acute phase reactant (11). Thus, for the patients in the acute or exacerbated phase, it was expected that MPV would decrease since the disease was in its inflammatory phase. However, for those who were in the stable condition of the disease, we expected that this would increase due to the dominance of the prethrombotic phase (12). In our study, even though the patients were in the exacerbated and inflammatory phase, the mean increase of MPV in the dead patients were reported higher than those who had survived. There were two possibilities: the first could be due to the increase of thrombotic events in the critically ill patients. The platelets aggregation followed by the release of mediators caused vascular endothelial damage, in which this vascular damage was eventually thrombogenic. Therefore, this increase indicated the dominance of the thrombotic phase of the disease. The second possible cause could be due to pulmonary hypertension which was reported to be higher in the dead patients than those who survived. The exact cause for MPV increase in the patients with pulmonary hypertension was unknown. However, hypoxia could lead to the release of larger platelets from the bone marrow (12). Therefore, the patients who had an increased MPV, especially those who had higher mPAP, could have a higher mortality rate.

Like MPV, the increase in the mean RDW also had a significant increase in-hospital mortality. Sedat Altin et al. showed that in the acute exacerbation of COPD, higher RDW levels at discharge were associated with a worse long-term outcome. Therefore, increase in this factor could also have a prognostic effect in exacerbated COPD patients. Özguil et al. also showed in their study. Two important causes which led to this increase were pulmonary hypertension followed by right ventricular failure and erythrocytosis inhibition due to severe inflammation $(16,18)$.

There was a significant difference in leukocytosis and neutrophilia between the dead patients and those who survived, which may be due to the inflammatory reactions. 
This increase in neutrophils was also observed in the exacerbated phase of COPD compared to the stable phase $(17,20)$. Donaldson et al. showed that in COPD patients, airway and systemic inflammatory markers increase over time; high levels of these markers are associated with a faster decline in lung function. The more the neutrophilia, the faster the decline the FEV1 percentage and lung function became (21). This neutrophilic left shift especially along with RDW could be helpful in determining the prognosis of the exacerbated disease. Furthermore, the FEV1 percentage of those who died was reported to be less than the FEV1 percentage in those who survived. The reason for this decrease can be interpreted by the increase in acute phase reactants since the percentage decreases with the increase of leukocytosis and neutrophilia, leading to pulmonary malfunction $(16,17,22)$. In this study, leukocytosis, and neutrophilia, along with the decrease in FEV1 percentage, had a prognostic effect in predicting the exacerbated disease.

As COPD progresses and the severity of the disease increases and the pulmonary function decreases, the mPAP increases (23). This increase in pressure not only causes an increase in the number of periods of the disease, but also leads to a decrease in the survival rate of these patients (24). There were some associated factors with pulmonary hypertension such as hypopnea, polycythemia, hypoxia, metabolic alkalosis, left ventricle failure and cachexia (25). Some studies confirmed that mean Pulmonary Artery Pressure (mPAP) is an independent risk factor for hospital mortality (26-28) which was emphasized in our study.

One of the limitations of our study was the fact that it was done in a unicenter. But there were some advantages that eliminate this limitation. This center was the only referral center for internal medicine patients in the southeast region of Iran with extremely high prevalence of COPD patients and large sample size. Further, limiting applicability were the spirometric studies and echocardiography not traditionally performed in ED. Moreover, patients who were unwilling to cooperate or had a history of confounding diseases were eliminated from the study.

\section{Conclusion}

In this study, the value of $\mathrm{CBC}$ and its related tests, especially WBC along with PMN, MPV, and RDW were very applicable in determining the patients' need for ICU admission as well as the prediction of hospital mortality. Based on this study, leukocytosis and neutrophilia, as well as the increase in MPV and RDW, were considerable factors in COPD patients, which emphasize the influence of severe inflammation and thrombotic events on the high mortality rate of these patients. Since thrombotic and inflammatory risk factors were influential in COPD patients' outcome, it could be possible to predict these patients' outcome by using a $\mathrm{CBC}$ and its related tests, though other important risk factors such as pulmonary hypertension and reduced FEV1 should be considered.

\section{Acknowledgment}

We are grateful to all those who helped and supported us to write the manuscript.

\section{Conflict of Interest}

Authors declared no conflict of interests.

\section{Funding}

This study was supported by Kerman University of Medical Sciences, Kerman, Iran.

\section{References}

1. Steiropoulos P, Papanas N, Nena E, et al. Mean platelet volume and platelet distribution width in patients with chronic obstructive pulmonary disease the role of comorbidities. Angiol. 2013; 64(7): 535-9. [DOI:10.1177/0003319712461436] [PMID]

2. Kucukardali Y, Onem Y, Terekeci H, Tangi F, Sahan B, Erikci A. Mean platelet volume (MPV) in intensive care unit (ICU) patients: is it a useful parameter in assessing prediction for mortality? J Med Med Sci. 2010; 1(3): 61-4.

3. Colkesen Y, Muderrisoglu H. The role of mean platelet volume in predicting thrombotic events. Clin Chem Lab Med. 2012; 50(4): 631-4. [DOI:10.1515/cclm.2011.806] [PMID]

4. Taylan M, Demir M, Kaya H, et al. Alterations of the neutrophil-lymphocyte ratio during the period of stable and acute exacerbation of chronic obstructive pulmonary disease patients. Clin Respir J. 2017; 11(3): 311-7. [DOI:10.1111/cri.12336] [PMID]

5. Turfan M, Erdogan E, Ertas G, et al. Usefulness of mean platelet volume for predicting stroke risk in atrial fibrillation patients. Blood Coagul Fibrinolysis. 2013; 24(1): 55-8. [DOI:10.1097/MBC.0b013e32835a0850] [PMID]

6. Manchanda J, Potekar R, Badiger S, Tiwari A. The study of platelet indices in acute coronary syndromes. Ann Pathol Lab Med. 2015; 2(1): A30-A5.

7. Beyazit Y, Sayilir A, Torun S, et al. Mean platelet volume as an indicator of disease severity in patients with acute pancreatitis. Clin Res Hepatol Gastroenterol. 2012; 36(2): 162-8. [DOI:10.1016/j.clinre.2011.10.003] [PMID]

8. Yuri Gasparyan A, Ayvazyan L, P Mikhailidis D, D Kitas G. Mean platelet volume: a link between thrombosis and inflammation? Curr Pharm Des. 2011; 17(1): 47-58. [DOI:10.2174/138161211795049804] [PMID]

9. Kisacik B, Tufan A, Kalyoncu U, et al. Mean platelet volume (MPV) as an inflammatory marker in ankylosing spondylitis and rheumatoid arthritis. Joint Bone Spine 2008; 75(3): 2914. [DOI:10.1016/j.jbspin.2007.06.016] [PMID]

10. Biljak VR, Pancirov D, Čepelak I, Popović-Grle S, Stjepanović G, Grubišić TŽ. Platelet count, mean platelet volume and smoking status in stable chronic obstructive pulmonary disease. Platelets 2011; 22(6): 466-70. [DOI:10.3109/09537104.2011.573887] [PMID] 
11. Ulasli SS, Ozyurek BA, Yilmaz EB, Ulubay G. Mean platelet volume as an inflammatory marker in acute exacerbation of chronic obstructive pulmonary disease. Pol Arch Med Wewn. 2012; 122(6): 284-90. [DOI:10.20452/pamw.1284]

12. Bansal R, Gupta HL, Goel A, Yadav M. Association of increased platelet volume in patients of chronic obstructive pulmonary disease: clinical implications. J Indian Acad Clin Med 2002; 3(2): 169-72.

13. Tertemiz KC, Alpaydin AO, Salman S, Ergan B, Cimrin AH Can red blood cell morphology be an indicator for COPD exacerbations? Eur Respir J. 2014; 44: 573.

14. Sevinc C, Tertemiz KC, Alpaydin AO, Ellidokuz H, Acara $\mathrm{C}$, Cimrin AH. Correlation between red cell distribution width and disease severity and mortality in COPD patients. Eur Respir J. 2014; 44: P520.

15. Seyhan E, Sökücü S, Ömür I. Usefulness of admission red cell distribution width as a predictor of early mortality in patients with severe exacerbation of chronic obstructive pulmonary disease. Eur Respir J. 2013; 42: 2158.

16. Altin S, Seyhan EC, Sökücü S, Tural S, Tural I, Günlüoglu G. Relation of red blood cell distribution width with longterm survival in acute exacerbation of chronic obstructive pulmonary disease. Eur Respir J. 2011; 38: 557.

17. Günay E, Ulaşlı SS, Akar O, et al. Neutrophil-to-lymphocyte ratio in chronic obstructive pulmonary disease: a retrospective study. J Inflamm. 2014; 37(2): 374-80. [DOI:10.1007/s10753-013-9749-1] [PMID]

18. Özgül A, Seyhan E, Ömür I. Red blood cell distribution with in patients with chronic obstructive pulmonary disease. Eur Respir J. 2013; 42: 1863.

19. Bursac Z, Gauss CH, Williams DK, Hosmer DW. Purposeful selection of variables in logistic regression. Source Code Biol Med. 2008; 3: 217. [DOI:10.1186/17510473-3-17] [PMID] [PMCID]
20. Kurtipek E, Bekci TT, Kesli R, Erdem SS, Terzi Y. The role of neutrophil-lymphocyte ratio and platelet-lymphocyte ratio in exacerbation of chronic obstructive pulmonary disease. $\mathrm{J}$ Pak Med Assoc. 2015; 65(12): 1283-7.

21. Donaldson GC, Seemungal TA, Patel IS, et al. Airway and systemic inflammation and decline in lung function in patients with COPD. Chest. 2005; 128(4): 1995-2004. [DOI:10.1378/chest.128.4.1995] [PMID]

22. Gan WQ, Man S, Senthilselvan A, Sin D. Association between chronic obstructive pulmonary disease and systemic inflammation: a systematic review and a meta-analysis. Thorax. 2004; 59(7): 574-80. [DOI:10.1136/thx.2003.019588] [PMID] [PMCID]

23. Cuttica MJ, Kalhan R, Shlobin OA, et al. Categorization and impact of pulmonary hypertension in patients with advanced COPD. Respir Med. 2010; 104(12): 1877-82. [DOI:10.1016/j.rmed.2010.05.009] [PMID]

24. Chaouat A, Naeije R, Weitzenblum E. Pulmonary hypertension in COPD. Eur Respir J. 2008; 32(5): 1371-85. [DOI:10.1183/09031936.00015608] [PMID]

25. Fekri MS, Torabi M, Shoul SA, Mirzaee M. Prevalence and predictors associated with severe pulmonary hypertension in COPD. Am J Emerg Med. 2018; 36(2): 277-80. [DOI:10.1016/j.ajem.2017.08.014] [PMID]

26. McGhan R, Radcliff T, Fish R, Sutherland ER, Welsh C, Make B. Predictors of rehospitalization and death after a severe exacerbation of COPD. Chest. 2007; 132(6): 1748-55. [DOI:10.1378/chest.06-3018] [PMID]

27. Holguin F, Folch E, Redd SC, Mannino DM. Comorbidity and mortality in COPD-related hospitalizations in the United States, 1979 to 2001. Chest. 2005; 128(4): 2005-11. [DOI:10.1378/chest.128.4.2005] [PMID]

28. Terzano C, Conti V, Di Stefano F, et al. Comorbidity, hospitalization, and mortality in COPD: results from a longitudinal study. Lung 2010; 188(4): 321-9. [DOI:10.1007/s00408-009-9222-y] [PMID]

\section{How to Cite This Article:}

Torabi M, Samareh Fekri M, Azizi Shoul S, Naghibzadeh Thahami A, Moeinaddini S. Evaluation of Prognostic Factors of Mortality in Patients with Chronic Obstructive Pulmonary Disease . J Adv Med Biomed Res. 2018; $26(118): 28-33$

Download citation:

$\underline{\text { BibTeX }}|\underline{\text { RIS }}| \underline{\text { EndNote }}|\underline{\text { Medlars }}| \underline{\text { ProCite }}|\underline{\text { Reference Manager }}| \underline{\text { RefWorks }}$

Send citation to:

(3) Mendeley 2 Zotero (0) RefWorks RefWorks 